\title{
QUANTUM PARABOLIC SOMBRERO
}

\author{
Ye. Hakobyan ${ }^{1}$, S. Ter-Antonyan ${ }^{2}$, V. Ter-Antonyan ${ }^{3}$ \\ Bogoliubov Laboratory of Theoretical Physics \\ Joint Institute for Nuclear Research \\ Dubna, Moscow Region, 141980, Russia
}

\begin{abstract}
We have discussed the energy levels and probability distribution density for a quantum particle placed in the two-dimensional sombrero-shaped potential $V\left(\rho, \rho_{0}\right)=\mu \omega^{2}\left|\rho^{2}-\rho_{0}^{2}\right| / 2$.
\end{abstract}

PACS: 02.60.+y; 03.65.Ge; 11.30.Qc

Keywords: Spectroscopy; Symmetry Breaking; Oscillator

\footnotetext{
${ }^{1}$ e-mail: yera@thsun1.jinr.ru

${ }^{2}$ e-mail: simonter@hotmail.com

${ }^{3}$ e-mail: terant@thsun1.jinr.ru
} 


\section{Introduction}

It is well known [1] that potentials having the form of a sombrero are convenient for explanation of spontaneous breaking of continuous symmetry.

On the other hand, until now there is no quantitative analysis for the motion of a quantum particle in this potential. The cause seems to be in that the sombrero, as a smooth potential, is a polynomial of the forth power, and for that potential the Schröedinger equation is not exactly solvable. However, there is no contradiction in refusing the smoothness condition and constructing a sombrero by means of the quadratic potential $W\left(\rho, \rho_{0}\right)=\mu \omega^{2}\left(\rho-\rho_{0}\right)^{2} / 2$, where $\rho=\sqrt{x^{2}+y^{2}}$ and $\rho_{0} \in[0, \infty)$ is a parameter. The one-dimensional version of the potential $W\left(\rho, \rho_{0}\right)$ is known as a double-oscillator [2]. The smoothness is broken for $W\left(\rho, \rho_{0}\right)$ at the point $\rho=0$. Nevertheless, the potential $W\left(\rho, \rho_{0}\right)$ is still complicated as the corresponding radial equation includes along with the terms $\rho^{2}$ and $\rho^{-2}$ the linear term $\rho$. This type of equations is not exactly solvable. One can go further and break the smoothness condition not at one but at an infinite number of points. The simplest possibility is realized by the potential $V\left(\rho, \rho_{0}\right)=\mu \omega^{2}\left|\rho^{2}-\rho_{0}^{2}\right| / 2$, which we call the parabolic

sombrero. Here, the smoothness is broken along the circle of the radius $\rho_{0}$. For $y=0$ (or $x=0$ ) the parabolic sombrero transforms into a two-center oscillator considered in paper [3]. The aim of the present article is to calculate the energy spectrum and wave functions of a quantum particle placed in the parabolic sombrero potential.

\section{Spectral Equation}

The Hamiltonian of the parabolic sombrero has the form

$$
H\left(\rho, \rho_{0}\right)=-\frac{\hbar^{2}}{2 \mu}\left(\frac{\partial^{2}}{\partial \rho^{2}}+\frac{1}{\rho} \frac{\partial}{\partial \rho}+\frac{1}{\rho^{2}} \frac{\partial^{2}}{\partial \varphi^{2}}\right)+\frac{\mu \omega^{2}}{2}\left|\rho^{2}-\rho_{0}^{2}\right|,
$$

where $\rho$ and $\phi$ are polar coordinates of the particle: $0 \leq \rho<\infty, 0 \leq \varphi<2 \pi$. To this Hamiltonian there correspond two radial equations

$$
\begin{gathered}
\frac{d^{2} R_{\text {in }}}{d r^{2}}+\frac{1}{r} \frac{d R_{\text {in }}}{d r}+\left(\frac{r^{2}}{4}-\frac{m^{2}}{r^{2}}-\xi_{\text {in }}\right) R_{\text {in }}=0, \\
\frac{d^{2} R_{\text {out }}}{d r^{2}}+\frac{1}{r} \frac{d R_{\text {out }}}{d r}-\left(\frac{r^{2}}{4}+\frac{m^{2}}{r^{2}}+\xi_{\text {out }}\right) R_{\text {out }}=0,
\end{gathered}
$$

where $r=(2 \mu \omega / \hbar)^{1 / 2} \rho, r_{0}=(2 \mu \omega / \hbar)^{1 / 2} \rho_{0}, \varepsilon=E /(\hbar \omega)$ and $m \in \mathbf{Z}$ is the eigenvalue of the angular momentum operator $\hat{L}=-i \partial / \partial \varphi$,

$$
\xi_{\text {in }}=r_{0}^{2} / 4-\varepsilon, \quad \xi_{\text {out }}=-r_{0}^{2} / 4-\varepsilon .
$$

The first equation is for the inner region $\left[0, r_{0}\right)$ of the sombrero, while the second one is for its outer $\left(r_{0}, \infty\right)$ region. We are interested in the solutions finite at $r=0$ 
and vanishing as $r \rightarrow \infty$. These conditions select the functions

$$
\begin{aligned}
& R_{\text {in }}(r)=C_{\text {in }} r^{|m|} e^{-i r^{2} / 4} F\left(\alpha, \gamma ; i r^{2} / 2\right), \\
& R_{\text {out }}(r)=C_{\text {out }} r^{|m|} e^{-r^{2} / 4} \Psi\left(a, \gamma ; r^{2} / 2\right) .
\end{aligned}
$$

Here $F$ and $\Psi$ are two independent solutions of the confluent hypergeometric equation:

$$
\begin{gathered}
F(\alpha, \gamma ; z)=1+\frac{\alpha}{\gamma} \frac{z}{1 !}+\frac{\alpha(\alpha+1)}{\gamma(\gamma+1)} \frac{z^{2}}{2 !}+\ldots, \\
\Gamma(a) \Psi(a, b ; z)=\int_{0}^{\infty} e^{-z t} t^{a-1}(1+t)^{b-a-1} d t \\
\alpha=\frac{|m|+1-i \xi_{\text {in }}}{2}, \quad a=\frac{|m|+1+\xi_{\text {out }}}{2}, \quad \gamma=|m|+1,
\end{gathered}
$$

and $C_{\text {in }}$ and $C_{\text {out }}$ are normalization constants. The formulae given below from the theory of confluent hypergeometric equation are taken from the monograph [4].

Let us require the equality of the logarithmic derivatives of the functions $R_{\text {in }}$ and $R_{\text {out }}$ at the point $r=r_{0}$. This condition works for $0<r_{0}<\infty$. For $r_{0}=0$ the smoothness at $r=r_{0}$ is observed just for $R_{\text {in }}$ and $R_{\text {out }}$, but not their derivatives (see for example [5]). After using formulae

$$
\begin{aligned}
F^{\prime}(\alpha, \gamma ; z) & =\frac{\alpha}{\gamma} F(\alpha+1, \gamma+1 ; z), \\
\Psi^{\prime}(\alpha, \gamma ; z) & =-a \Psi(a+1, \gamma+1 ; z),
\end{aligned}
$$

we come to the spectral equation

$$
\frac{i \alpha}{\gamma} \frac{F\left(\alpha+1, \gamma+1 ; i z_{0}\right)}{F\left(\alpha, \gamma ; i z_{0}\right)}-\frac{i}{2}=-a \frac{\Psi\left(a+1, \gamma+1 ; z_{0}\right)}{\Psi\left(a, \gamma ; z_{0}\right)}-\frac{1}{2},
$$

where $z_{0}=r_{0}^{2} / 2$.

Note that from the Kummer transformation

$$
F(\alpha, \gamma ; z)=e^{z} F(\gamma-\alpha, \gamma ;-z)
$$

the relation $\gamma-\alpha^{*}=\alpha$ and the recurrent formula

$$
(\alpha-\gamma) F\left(\alpha, \gamma+1 ; i r_{0}^{2} / 2\right)=-\gamma F\left(\alpha, \gamma ; i r_{0}^{2} / 2\right)+\alpha F\left(\alpha+1, \gamma+1 ; i r_{0}^{2} / 2\right)
$$

there follows that the left hand side of the spectral equation is real. From the Kummer transformation there follows that the function $R_{i n}$ is also real.

In the next section, we will discuss the results obtained from the spectral equation by numerical calculations. 


\section{$3 \quad$ Energy Levels}

For $r_{0}=0$ the parabolic sombrero transforms into the circular oscillator for which the state is determined by the quantum numbers $\left(n_{r}, m\right)$, and the $n$-th energy level is given by the formula $\varepsilon=n+1$ and has multiplicity $g_{n}=n+1\left(n_{r}\right.$ is the number of zeroes of the radial wave function in the region $\left.(0, \infty), n=2 n_{r}+|m|\right)$.

The inclusion of the parameter $r_{0}$ splits the energy levels and transforms them into an infinite set of intersecting lines in the plane $\left(\varepsilon, r_{0}\right)$ composing a complicated picture (see Fig. 1).

Let us separate the energy lines of the parabolic sombrero into clusters of three types: with fixed $n$ (n-cluster), $|m|\left(|m|\right.$-cluster) and $n_{r}\left(n_{r}\right.$-cluster).

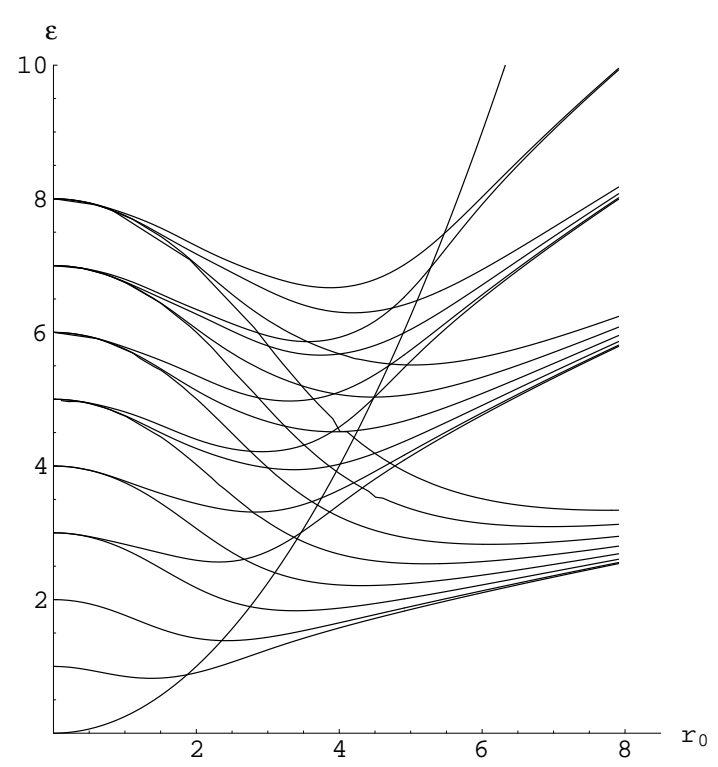

Fig. 1: Dependence of the energy levels of the parabolic sombrero on $r_{0}$. Here as well as in Figs. 2-5 the parabola describes the parabolic sombrero potential as a function of $r_{0}$ for $r=0$.

a. The $n$-cluster possesses $(n / 2+1)$ or $(n+1) / 2$ lines for even and odd $n$, respectively (see Fig. 2). For large values of the parameter $r_{0}$, the lines of the $n$-cluster are very much separated from each other. With decreasing parameter $r_{0}$ the distance between the lines of the $n$-cluster decreases. Beginning at some $r_{0}$, particular for every line, the lines jump above the top of the parabolic sombrero and with the following decrease of $r_{0}$ the lines combine in one $(n+1)$ degenerate energy level of a circular oscillator.

b. Every $|m|$-cluster includes an infinite number of lines (see Fig. 3). With growth of the parameter $r_{0}$, each line of the $|m|$-cluster, located above the top of the sombrero, first slightly lowers, then grows and starting from some parameter $r_{0}$, particular for each line, is captured by a circular wall. For further growth of $r_{0}$ 

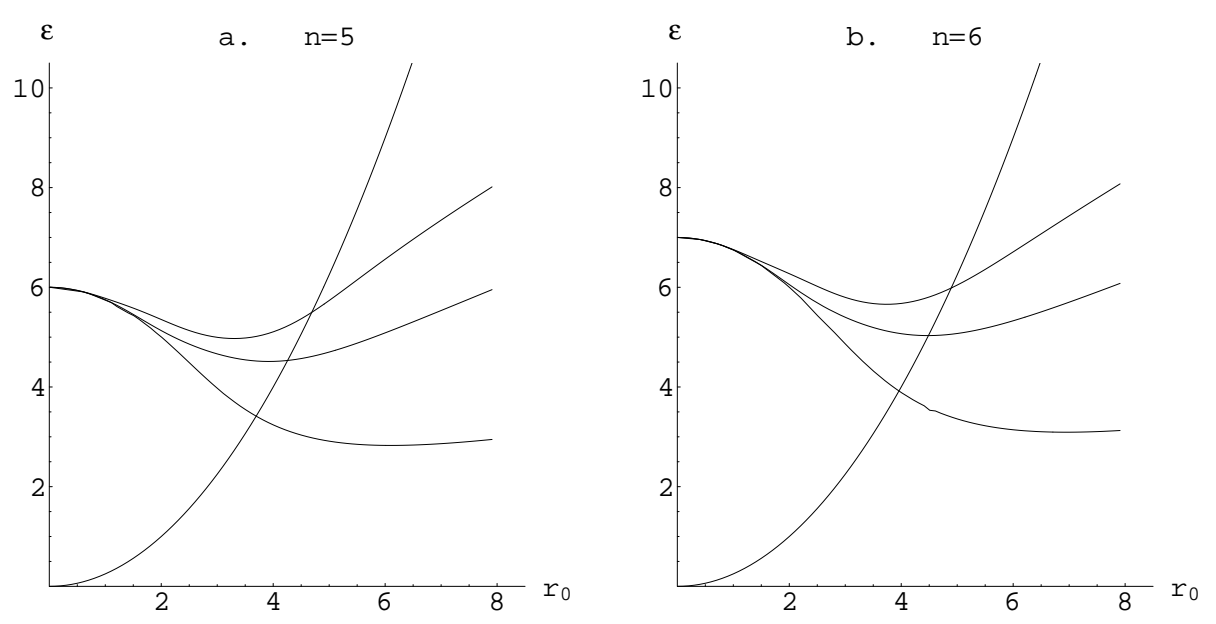

Fig. 2: Dependence of the lines of $n$-clusters on the parameter $r_{0}$ for $n=5$ and $n=6$. To larger values of $\varepsilon\left(r_{0}\right)$ (for fixed $r_{0}$ ) corresponds the larger $n_{r}$.

the lines of the $|m|$-cluster grow with different velocity: the more $n_{r}$ the faster the growth of the line.

c. Let us compare the lines of $|m|$-cluster with $m=0$ and the energy levels of the two-center quantum oscillator [3]. The equation describing the two-center oscillator can not be obtained from radial equation of the parabolic sombrero: if we substitute $m=0$ and $R=f / r^{1 / 2}$ into the radial equation, we eliminate the centrifugal potential $\mathrm{m}^{2} / \mathrm{r}^{2}$ and delete the term with first derivative, but then arises an additional centrifugal potential. This new centrifugal potential influences the energy spectrum, as the result of which the spectroscopy of the parabolic sombrero with $m=0$ and the spectroscopy of the two-center oscillator are not identical. As we can see in Fig. 4, with the increase in the parameter $r_{0}$ the energy levels of the two-center quantum oscillator merge in pairs and between them is the line corresponding to the parabolic sombrero.

d. Quite interesting is the behavior of the lines of $n_{r}$-clusters (see Fig. 5). Every $|m|$-cluster, as well as $n_{r}$-cluster, includes an infinite number of lines. With the growth of the parameter $r_{0}$, the lines of the $n_{r}$-cluster are gradually coming together, then captured by the circular wall, and continuing the approach merge into one line. Thus, for $r_{0} \rightarrow \infty$ we have an infinite number of levels, each being an infinite degenerated $n_{r}$-cluster.

e. Using the known formula [5]

$$
\frac{\partial E_{n_{r}, m}}{\partial \rho_{0}}=\left(\frac{\partial \hat{H}}{\partial \rho_{0}}\right)_{n_{r}, m ; n_{r}, m},
$$



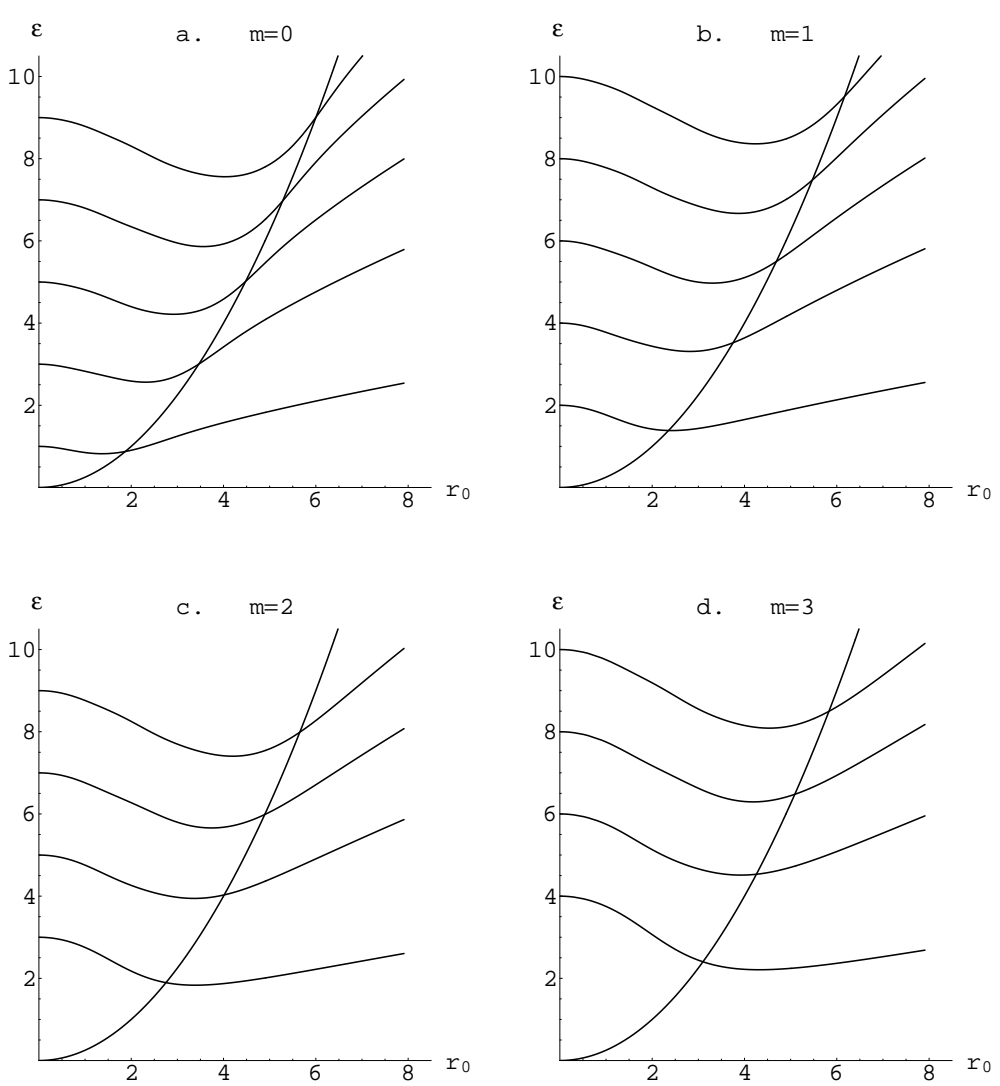

Fig. 3: Dependence of the lines of $|m|$-clusters on the parameter $r_{0}$ for $m=0,1,2,3$.

we obtain

$$
\frac{\partial E_{n_{r}, m}}{\partial \rho_{0}}=\mu \omega^{2} \rho_{0}\left[2 \int_{0}^{\rho_{0}}\left(R_{n_{r}, m}^{i n}\right)^{2} \rho d \rho-1\right] .
$$

The integral in the brackets is a monotonously increasing function of $\rho_{0}$ with the range of values $[0,1]$. Expanding it in powers of $\rho_{0}$ (for small $\rho_{0}$ ) and $1 / \rho_{0}$ (for large $\left.\rho_{0}\right)$, we obtain

$$
\begin{gathered}
\varepsilon_{n_{r}, m}\left(r_{0}\right) \simeq 2 n_{r}+|m|+1-\frac{r_{0}^{2}}{4}, \quad \text { for } \quad r_{0} \ll 1, \\
\varepsilon_{n_{r}, m}\left(r_{0}\right) \simeq \frac{r_{0}^{2}}{4}-A_{n_{r}, m} r_{0}, \quad \text { for } \quad r_{0} \gg 1,
\end{gathered}
$$

where $A_{n_{r}, m}$ is the matrix with positive elements independent of $r_{0}$. As will be shown below (see Conclusion), for extremely large values of the parameter $r_{0}$, the quantities $A_{n_{r}, m}$ cease to depend on the quantum number $m$. 


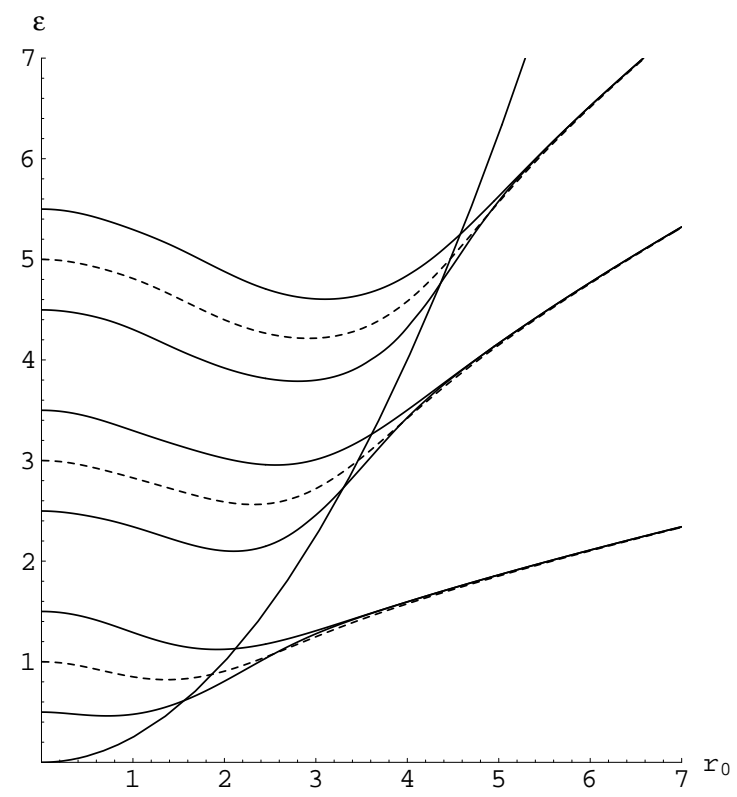

Fig. 4: Comparison of the lines of the " $m=0$ "-cluster of the parabolic sombrero with the energy levels of the two-center quantum oscillator taken from [3]. Solid lines correspond to the two-center quantum oscillator; dotted lines to the parabolic sombrero.

We come to the following conclusions: firstly, the corrections to the spectrum of the circular oscillator $\left(r_{0} \ll 1\right)$ are not linear but quadratic in $r_{0}$; secondly, after the capture by the wall the energy levels accumulate near the top of the sombrero forming a ring with thickness $r_{0}$ times less than the top of the sombrero. The last conclusion is confirmed by Fig. 5 .

\section{Probability Distribution}

Let us introduce two functions

$$
\begin{gathered}
D_{\text {in }}(r)=r^{|m|} e^{-i r^{2} / 4} F\left(\alpha, \gamma ; i r^{2} / 2\right), \\
D_{\text {out }}(r)=r^{|m|} e^{-r^{2} / 4} \Psi\left(a, \gamma ; r^{2} / 2\right),
\end{gathered}
$$

and rewrite the functions $R_{\text {in }}$ and $R_{\text {out }}$ in the form

$$
R_{\text {in }}(r)=C_{\text {in }} D_{\text {in }}(r), \quad R_{\text {out }}(r)=C_{\text {out }} D_{\text {out }}(r) .
$$

To find the normalization constants, we just sew together $R_{\text {in }}$ and $R_{\text {out }}$ at the point $r=r_{0}$ :

$$
C_{\text {in }} D_{\text {in }}(r)=C_{\text {out }} D_{\text {out }}(r),
$$



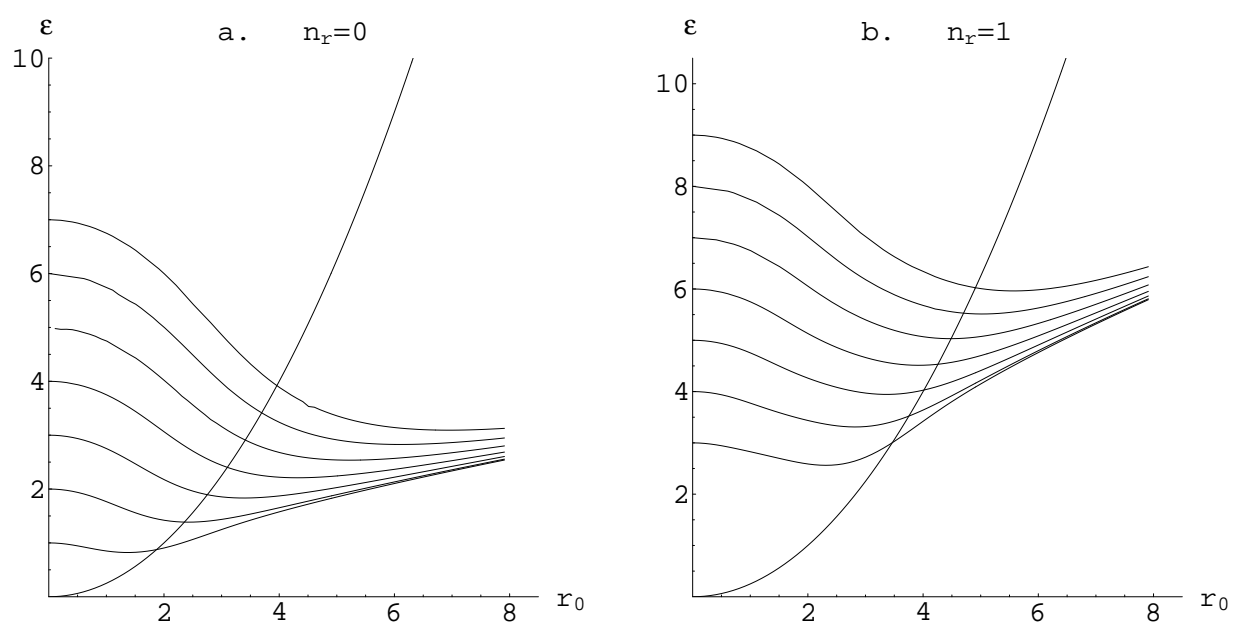

Fig. 5: Dependence of the lines of $n_{r}$-clusters of the parabolic sombrero on the parameter $r_{0}$ for $n_{r}=0,1$.

and demand

$$
C_{\text {in }}^{2} \int_{0}^{r_{0}} D_{\text {in }}^{2} r d r+C_{\text {out }}^{2} \int_{r_{0}}^{\infty} D_{\text {out }}^{2} r d r=1
$$

It is easy to conclude from these two equations that

$$
C_{\text {in }}=D_{\text {out }}\left(r_{0}\right) / Q\left(r_{0}\right), \quad C_{\text {out }}=D_{\text {in }}\left(r_{0}\right) / Q\left(r_{0}\right)
$$

where

$$
Q\left(r_{0}\right)=\left[D_{\text {out }}^{2}\left(r_{0}\right) \int_{0}^{r_{0}} D_{\text {in }}^{2}(r) r d r+D_{\text {in }}^{2}\left(r_{0}\right) \int_{r_{0}}^{\infty} D_{\text {out }}^{2}(r) r d r\right]^{\frac{1}{2}}
$$

The obtained formulae allow one to look at the picture of motion of the energy levels from the point of view of probability distribution. The diagrams of numerical calculations for the functions $r R^{2}$ for $n_{r}=3, m=0$ and different values of the parameter $r_{0}$ are presented in Fig. 6. Considering these diagrams in ascending order of the parameter $r_{0}$ we see that they confirm the general scenario of the motion of the levels described in the previous section.

Fig. 6a corresponds to the circular oscillator. In Fig. 6b the moment of capture of the level by the wall $\left(r_{c}\right.$ is the value of the parameter $r_{0}$ when the capture takes place) is drawn. Figs. 6c and 6d demonstrate the shift of the particle together with the wall far from the origin of the coordinates with further increase in the parameter $r_{0}$. 

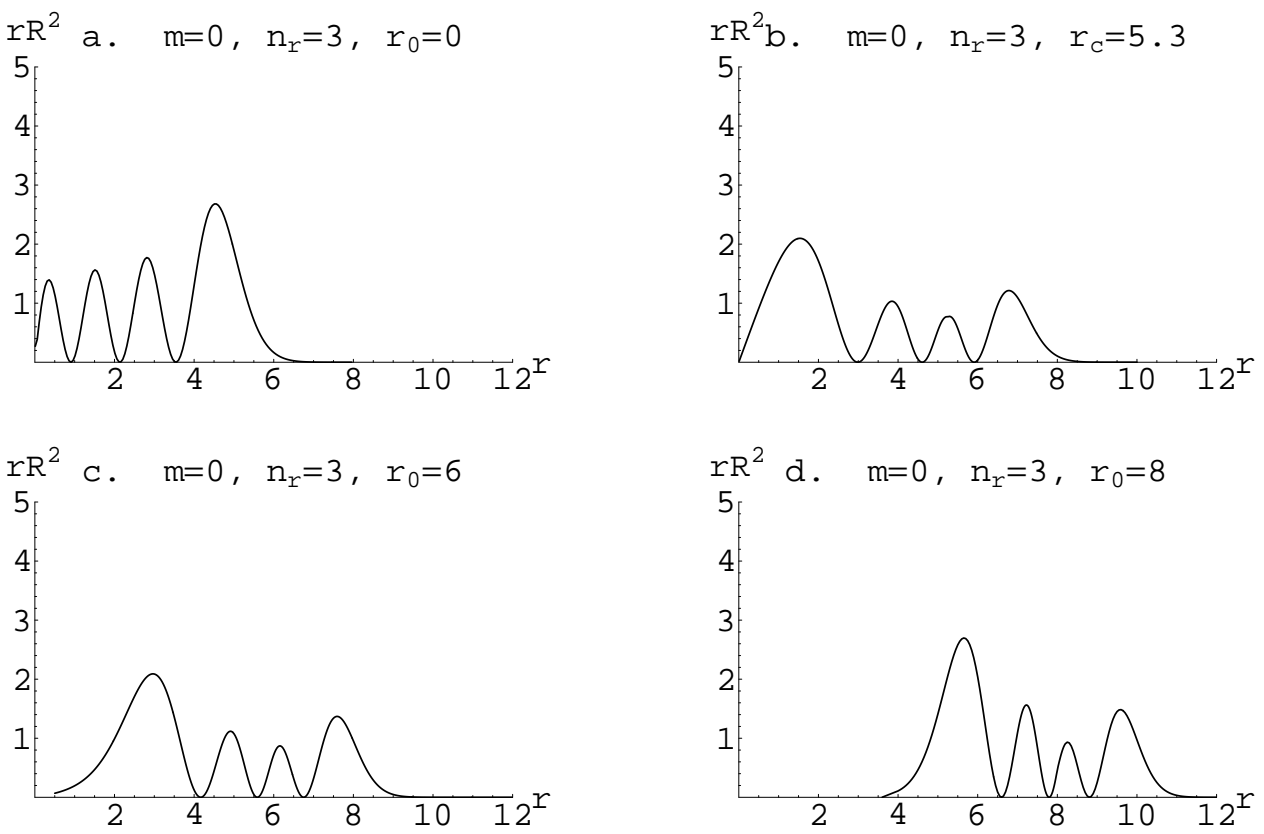

Fig. 6: Diagrams of the probability distributions for $m=0, n_{r}=3$ and different values of $r_{0}$.

\section{Conclusion}

In conclusion, let us discuss the limit $r_{0} \rightarrow \infty$ more thoroughly. As $r_{0} \rightarrow \infty$ the height of the barrier $\left(r_{0}^{2} / 4\right)$ increases, and that is why the wave functions, corresponding to the energy levels in the wall differ from zero only in the region of large $r$, where the centrifugal term $m^{2} / r^{2}$ could be neglected. In such an approach the functions $R_{\text {in }}$ and $R_{\text {out }}$ as well as the energy levels cease depending on quantum number $m$, which in its turn indicates the dependence (in the limit of large $r_{0}$ ) of the spectroscopy of captured levels practically on the quantum number $n_{r}$ only. Such a conclusion is in agreement with the tendency, presented in Fig. 5, and with the general philosophy of spontaneous breaking of continuous global symmetry [1].

\section{Acknowledgment}

The authors would like to thank G. Pogosyan and A. Sissakian for fruitful discussions. The work of Ye. Hakobyan was partially supported by Russian Foundation for Basic Research (RFBR) under the project \# 98-01-00330. 


\section{References}

[1] A. Sudbery, Quantum Mechanics and Particles of Nature (Cambridge University Press, Cambridge, 1986).

[2] E. Merzbacher, Quantum Mechanics (John Wiley \& Sons, New York, 1970).

[3] A. Sissakian, S. Ter-Antonyan, V. Ter-Antonyan, JINR Preprint P2-92-514 (Dubna, 1992).

[4] A. Erdélyi, W. Magnus, F. Oberhettinger and F.G. Tricomi (eds), Higher Transcendental Functions, Vol. 1 (McGraw Hill, New York, 1953).

[5] L.D. Landau and E.M. Lifshitz, Quantum Mechanics, Nonrelativistic Theory (Pergamon Press, Oxford, 1965). 